\title{
Editorial
}

\section{Water Tariffs and Affordability: The Economics and Policy of Protecting the Poor}

\author{
Joseph Cook* and Dale Whittington ${ }^{\dagger, *}$ \\ *School of Economic Sciences \\ Washington State University Pullman \\ WA 99164, United States \\ 'Department of Environmental Sciences and Engineering \\ and Department of City and Regional Planning \\ University of North Carolina at Chapel Hill \\ Chapel Hill, NC 27156, United States \\ Global Development Institute \\ University of Manchester \\ Manchester M13 9PL, United Kingdom
}

Published 31 July 2020

\section{Introduction}

We are writing the editorial note to this special issue on "Water Tariffs and Affordability: the Economics and Policy of Protecting the Poor" at a time (July 2020) when access to water and sanitation services is more essential than ever. Handwashing is a critical measure to prevent the spread of COVID-19, but access to reliable water and sanitation services (and soap) is far from universal for households and health care facilities across the Global South.

The genesis of the special issue on affordability predated the pandemic. A key strategy for adapting to changing water availability and rapid urbanization is a move towards full cost recovery tariffs for water and sanitation services. Volumetric prices that reflect the long-run marginal cost of supply help to incentivize customers to use scarce water resources wisely. Tariffs that recover the full costs of providing improved water and sanitation services also encourage managers to plan 
for future expansion by deploying capital resources wisely rather than relying on subsidies. Because these services are substantially underpriced in most places, this strategy implies that careful attention must be directed at programs to ensure that poor households can afford to pay their water bills. Achieving access to "safe and affordable" drinking water is target 6.1 of the United Nations' Sustainable Development Goals to "ensure access to water and sanitation for all". The SDGs include similar targets for goals in energy, transportation, housing, and education.

In the U.S., water and sewer tariffs have been increasing faster than inflation, ${ }^{1}$ in part to replace aging infrastructure. The public and policymakers are also increasingly focused on affordability. Many U.S. water utilities have "customer assistance programs" (CAPs) such as means-tested fixed bill reductions or conservation assistance programs (US EPA 2016). But these programs face challenges in increasing uptake and securing funding, particularly in places where crosssubsidies from other ratepayers are specifically prohibited. Some utilities are experimenting with different types of CAPs. For example, the city of Philadelphia recently introduced a new tariff structure for the poor that limits their water bills to 2-4\% of their income (Bouma 2018). In California, the State Water Board, responding to a 2015 mandate from the Legislature, recently submitted recommendations for a statewide water rate assistance program for low-income households (Pierce et al. 2020).

The COVID-19 pandemic has heightened these affordability concerns and accelerated experimentation with CAP programs. Though common in Europe before the pandemic (WAREG 2017), various types of moratoria on disconnections for non-payments were put in place in spring 2020 in 13 U.S. states and the District of Columbia (Campbell-Ferrari and Wilson 2020). Though there is no centralized database on CAPs, it appears that many U.S. utilities are offering deferred payment plans or expediting enrollment in existing assistance programs. ${ }^{2}$ Some governments in low- and middle-income countries are subsidizing water bills. In Vietnam, the government asked utilities to reduce water rates by $10 \%$ in rural areas and 5$10 \%$ in urban areas, for three months. ${ }^{3}$ Ghana's government agreed to provide free water in April, May and June to the entire country, supplying financial support to

\footnotetext{
${ }^{1}$ See https://www.circleofblue.org/waterpricing/.

${ }^{2}$ The city of Seattle, for example, offered an income-qualified Utility Discount Program before the pandemic. In response to COVID-19, the utility is allowing residents to self-certify income to qualify, rather than provide income documentation. See https://www.seattle.gov/utilities, accessed July 7, 2020.

${ }^{3}$ Personal communication with Prof. Pham Khanh Nam, July 3, 2020.
} 
service providers to compensate them for the revenue losses. ${ }^{4}$ They also pledged to provide free tanker water services to areas not served by network services.

These crisis responses will have long-term consequences on water utilities around the world. Stepping back from a focus only on the water and sanitation sector, the COVID-19 pandemic has resulted in both supply and demand shocks in many sectors of the economy. These have caused reductions in employment, production, and spending that have rippled through economies throughout the world. These reductions in economic activity have caused financial losses to most owners of capital and labor. Everyone is now negotiating to be reimbursed for their losses. For example, the unemployed are asking for payments from governments to make up for lost salary; airlines are asking governments for bailouts because no one wants to fly; and tenants are asking for rent holidays. Water utilities are also facing revenue shortfalls because some customers are unemployed and cannot pay their water bills. If water and sanitation services were unaffordable to some customers before the pandemic, they have become even more so now.

One can make a strong case for why water utilities and their customers need financial help during the COVID-19 pandemic. It obviously makes no sense to reduce water and sanitation services during a public health crisis. But other sectors can also make a strong case for public subsidies. Losses must be borne by someone, and during the COVID-19 pandemic, a key question for society is how these losses should be allocated. As a general observation, most people are probably less worried about market losses incurred by owners of capital (e.g. shareholders) than market losses allocated to labor (e.g., unemployed workers).

Can losses in the water sector, which is extremely capital intensive, be allocated to owners of capital rather than their workers or customers? Utilities that financed these capital expenditures with debt are now in a position to negotiate with those who hold their debt to postpone payments or even forgive a portion of the outstanding debt. However, this mechanism of allocating losses is not open to most water utilities in the Global South because they are publicly-owned and do not have much debt; their capital costs were paid by national governments or donors, not debt-financed. Their revenues are needed to pay operation and maintenance expenses, which are largely labor, electricity and chemicals.

If a utility's revenues from customers collapse due to the COVID-19 pandemic because customers cannot (or are not required to) pay their water bills, the utility has few expenses that can be cut without threatening the quality of the services it

${ }^{4}$ Daily Graphic (April 14, 2020). Free Water Directive to Cost Almost Ghs $300 \mathrm{~m}$. Available@ https://www.graphic.com.gh/news/general-news/free-water-directive-to-cost-almost-gh-300m.html. Accessed July 7, 2020. 
provides. So, how are publicly-owned water utilities in the Global South going to continue providing water and sanitation services if their customers do not pay their water bills? Because borrowing from the capital markets is very unlikely, there are only three main possibilities. First, higher-level governments and donors could provide funds, as is the case in Ghana. This is what water utility management and customers would like, but as noted, everyone is now asking for help covering their losses. Central governments, especially in the Global South, are under great financial pressure, and water utilities will not necessarily be at the head of the queue. Second, water utilities can reduce payments to labor. This option is politically unacceptable and may adversely affect the quality of services provided. The final option is to ask households who have not lost their jobs to continue to pay their water bills. If everyone is permitted to stop paying their water bills, utilities will face budget shortfalls that will be very hard to fill. We believe that water professionals should not jump from the fact that water and sanitation services need to be maintained during a pandemic to support a policy proposal that all customers need a "tariff holiday" - a period when no one has to pay their water bills. Instead, utilities can target assistance to households that really cannot afford to pay for water and sanitation services with the types of CAPs mentioned above.

The five papers in this special issue present new ideas and research results along many of these themes. How can utilities tailor programs to help those who struggle to pay their bills, either episodically or chronically? How should governments measure how much of a burden water and sewer bills are? How should affordability be measured in rural settings characterized by a multiplicity of water sources that households dynamically choose among with changes in weather and water quality? How do households themselves perceive the reasonableness of water bills? Are policies that protect forests and their watershed services "progressive” in that more of their benefits flow to areas with higher poverty rates?

\section{Overview of the Papers in this Special Issue}

\section{1. "Examining the economics of affordability through water diaries in coastal Bangladesh" by Hoque and Hope}

Current measurement tools are not well-equipped to assess affordability challenges in rural areas of the Global South where most households are not connected to piped networks. This is in part because the time costs of collecting water are often more important to households in these settings than the financial collection costs, particularly since most rural households do not regularly pay for water services. Hoque and Hope argue that the current treatment of affordability by international and national organizations simplifies the situation facing rural households for three 
reasons. First, unlike urban, connected households, rural households are more likely to collect from multiple sources. Measuring affordability by focusing only on the main or "dominant" source, as is standard, will be misleading. Second, seasonality and rainfall play a larger role, such that household decisions about what water sources to use are dynamic. Newer evidence of detailed handpump monitoring in African settings shows large fluctuations in water demand over time. When the rainy season begins, households are more likely to switch away from sources that require payment. Again, an affordability calculation based on an assumed fixed daily demand is likely to be flawed. The third difference is the heterogeneity in the quality of water sources, which they term the "environmental" dimension. Existing affordability calculations can mischaracterize households that spend a small amount of money or time collecting low-quality or unsafe water.

To illustrate the challenges of measuring affordability along these dimensions of dominance, time/seasonality, and environment, Hoque and Hope report results from a longitudinal study of water use in coastal Bangladesh. They had 120 households complete pictorial "water diaries" for 18 weeks. These diaries are a unique methodological approach to measuring the full extent of collection costs (time and financial). These diaries captured the types and locations of water sources used, the quantity collected, the financial costs of water, and information on who in the household collected water. Hoque and Hope pair analysis of these water diaries with a household survey and detailed information on other financial purchases. Their results show five main types of households. The first group have no financial expenditures on water. The second have spending that varies predictably with rainfall, demonstrating the importance of the "time" dimension of affordability. The third spends in an "ad hoc" fashion, with spending increasing for example during festivals. The final two categories are "low" and "high" expenditures. These two groups spend approximately $2 \%$ and $5 \%$ of all expenditures on water. Although these might be considered "affordable" by standard metrics, Hoque and Hope note that many of these customers are receiving low-quality water. Furthermore, these figures only capture recurring financial expenditures on water. Although the water diaries capture information on the time spent collecting water, the paper does not convert this time into a monetary value. Capital investments to cope with poor supply (e.g. storage tanks, water treatment devices) are similarly not captured.

In summary, Hoque and Hope call on water policy analysts to move beyond a focus only on prices and tariffs in affordability discussions. Rural water collection patterns are dynamic and mediated by weather, habits, groundwater quality and cultural norms. Increased inter-disciplinary and longitudinal research is needed to 
understand these patterns and assess the "affordability" challenges facing rural households, particularly the poorest and most marginalized. This type of research can also help inform policy to improve access to safely-managed water services in rural areas, including the design and delivery of infrastructure, management responsibilities, and pricing and payment modes to recover operation, maintenance and capital depreciation costs.

\section{2. "Measuring the affordability of water supply, sanitation, and hygiene services: A new approach" by Andres, Brocklehurst, Grabinsky, Joseph and Thibert}

The paper by Andres et al. represent another innovative approach to improve the way affordability is measured. The authors begin with a discussion of the key measurement challenges. This includes a dependence on self-reported household expenditure, which often does not reflect the actual costs of service to the provider and almost never includes initial capital costs. It also fails to distinguish between the type and level of service that a household receives (a key point in Hoque and Hope above), and depends on thresholds to determine affordability.

Andres et al. propose a new measurement approach building on one that looks at the total costs of a basket of WASH services. Their approach is characterized by five steps. First, the analyst defines a minimum basket of WASH services that policymakers can agree, in a given local context, that all households should be able to access. Thus an affordability calculation in a rural area might focus on access to protected boreholes while in an urban area it might focus on bills for customers with piped connections. Some households will currently have access to this level of service while others may not. Second, this minimum basket is costed using actual market-based prices and/or using the tariffs faced by the users. Importantly, the Andres et al. approach includes the initial capital costs required to access the service as well as recurring costs. Third, the analyst compares a household's total income/expenditure with data on its current level of WASH services. Fourth, the minimum household income/expenditure required to afford the minimum basket of WASH services is determined. Finally, the analyst presents results on affordability disaggregating households by access and affordability. In other words, some households in a given area may have access to the chosen basket of services (e.g. a piped connection) while others may not. By using a predetermined threshold, the analyst can choose to present a $2 \times 2$ matrix, as Andres et al. do, showing groups (a) with access to the basket for whom the service is affordable, (b) with access for whom the service is unaffordable, (c) without access and who would find it affordable if they could access it, and (d) without access and who would find it 
unaffordable if they were able to access it. Furthermore, their approach can be used to simulate the impact of various types of policy scenarios.

They demonstrate their approach in rural Nigeria using data from the 2015-16 Nigeria General Household Survey (NGHS). Assuming a protected borehole with a handpump within a 30 minute walk is the locally-appropriate, minimum acceptable level of service, they estimate both levelized capital costs and recurring operations and maintenance costs. They use data from the NGHS on access to this type of service and actual reported expenditures on water. Only $10 \%$ of households have improved water on premises, $47 \%$ have access to a basic water source within 30 minutes, and the remainder walk further. Reported expenditures are extremely low, so traditional affordability calculations would show that there is no problem: households spend, on average, only $0.29 \%$ of total expenditures on water. Using the authors' proposed approach that includes the full costs of service presents a different picture. Sixty percent of those with access to the basket currently would be able to afford the recurring costs but not the capital costs (which are assumed to be repaid over two years). They close by simulating affordability under a government program that would subsidize $80 \%$ of capital costs and $40 \%$ of recurring costs.

\section{3. "Households' perceptions of "reasonable" water bills in Ho Chi Minh City, Vietnam" by Thuy, Nam and Whittington}

The policy discussion about affordability has so far largely focused on assessing whether households are spending greater than a given threshold percentage of their income on water and sanitation services. If households spend more than, for example, $5 \%$ on their combined water and sewer bill then the services are "unaffordable". Thuy et al., as well as several earlier authors, note that these thresholds are challenging in part because they depend on the prices of other goods and services. Since "affordable" housing, health care, education (and others) are all targets of the Sustainable Development Goals along with water and sanitation services, finding "affordable" prices for services in isolation will be extremely difficult. Deciding what these thresholds (e.g. 3\%, 5\%) should be is also challenging and has largely been the domain of government policy or expert opinion, with little attempt to ask households what they consider to be "affordable" services. Similarly, tariff reforms require political support. Households who feel that existing or revised tariffs are "reasonable" or "fair" for their household's use are more likely to support reform. The sector knows relatively little about how customers perceive the reasonableness or fairness of various pricing policies or tariff levels.

Thuy et al. tackle these latter two challenges by directly asking 2075 households in Ho Chi Minh City (HCMC), Vietnam about "reasonable" bills. To counter 
status-quo effects, they randomly assign monthly quantities of water use to households. They elicit preferences with two approaches. The first is an openended question that essentially asks what the household considers a reasonable bill for the exogenously-assigned monthly water use (as well as their own current water use) if piped services in HCMC improved in quality and hours of service. A second set of households completed a "stochastic payment card" (SPC) that presented them with a set of nine prices for the assigned quantity and asked them to rate the reasonableness as "definitely not reasonable", "probably not reasonable", "not sure", "probably reasonable" or "definitely reasonable". The authors conservatively considered the reasonable bill as the highest bill the household characterized as "definitely reasonable". They then explore the correlation between reasonable bills and household characteristics with OLS regression. They also asked what households believed "reasonable" meant to them, and if they distinguished between "fair" bills and "reasonable" bills.

Although one might simplistically think that households always consider lower bills as "more reasonable", one of the most striking results is that nearly all households considered some bills to be unreasonably low. The vast majority (92\%) of respondents in the SPC approach followed a response pattern that included low bills that were considered "not reasonable", intermediate bill levels that were "reasonable" and high bill levels that households deemed again "not reasonable". Only one percent of respondents believed that lower bills were always more reasonable. Thuy et al. believe households are concerned that service providers recover enough costs to maintain or improve services. When asked what "reasonable" meant, one quarter of respondents mentioned one of the dimensions as "the bill is enough to cover the cost of provision". Using the open-ended response data on exogenously-assigned quantities, they find that reasonable bills are modestly larger than households' current bills, which might reflect the service level improvement in the hypothetical scenario. Customers' interpretation of "reasonableness" seem to include little emphasis on affordability to the poor; only $29 \%$ of respondents specifically mentioned that a reasonable bill was one that was affordable to poor households. The authors also find that reasonable water bills increase as the exogenously-assigned quantity of water increases. However, respondents do not think that it is reasonable for households to pay an increasing per unit charge if they use more water, i.e., water bills should not increase as a nonlinear function of quantity. Finally, Thuy et al. find that respondents' selfreported reasonable water bills suggest support for a positive fixed charge in the tariff structure. Respondents believed that there was a reasonable minimum water bill for a household even if it uses very little water during a month. 


\section{4. "Is the distribution of ecosystem service benefits pro-poor? evidence from water purification by forests in Thailand" by Vincent, Nabangchang and Shi}

Ecosystem service provision is another domain in which poor households may be disproportionately affected. As Vincent $e t$ al. discuss, it is commonly believed that the poor bear more of the losses when forest or other ecosystem services are degraded. There is, however, relatively little empirical evidence for this claim. This paper addresses this gap by examining the distribution of marginal benefits of forest protection (via lower water treatment costs) in Thailand.

The study first establishes the link between forest protection and short-run water treatment costs using 10 years of quarterly panel data for 158 urban water utilities (or "branches") in Thailand, covering a substantial portion of the country. Empirical evidence on this link is still relatively rare, particularly with the type of panel data used here. Because electricity prices are uniform across all branches and labor rates are similarly centralized, the time fixed effects absorb any variation in these (substantial) categories of water treatment costs. This leaves the empirical focus on chemicals used to treat the raw surface water; none of the utilities sourced from groundwater. Branch-level fixed effects control for differences across utilities in treatment costs caused by geology, total area, topography. The model therefore uses variation in land use (taken from national land use data) over time within a branch. Controlling for total water treated, rainfall, and capital stock (the number of treatment plants within one branch), Vincent et al. find that a $1 \%$ increase in agricultural land is associated with a $0.57 \%$ increase in chemical water treatment costs, in line with other existing studies. A 1\% increase in urban land cover is associated with a $0.28 \%$ increase in treatment costs.

If forest protection lowers chemical treatment costs, are these benefits more or less likely to accrue to poor households? Lacking household- or branch-level data on poverty, Vincent et al. rely on province-level poverty rates, which vary substantially. Using a variety of non-parametric approaches, they find no statistically significant relationship between predicted marginal benefits of forest protection and poverty rates. In other words, they find no evidence that this particular ecosystem service is either progressive or regressive in its benefits. They also note that the absolute value of the predicted marginal benefits are relatively small. The authors model the hypothetical impact of a large (26\%) increase in forest cover across all provinces assuming all chemical treatment costs savings are passed onto consumers via lower prices and using household-level water expenditure data from an earlier smaller-scale study. They find that such a program would reduce the percentage of income that the poorest quintile spend on water from $8.9 \%$ to $7.8 \%$. 
Finally, the authors note that although these gross benefits of water purification services are neither progressive nor regressive, the net benefits of forest protection may still be progressive if the costs of protecting forests are lower in higherpoverty provinces.

\section{5. "Choosing among pro-poor policy options in the delivery of municipal water services" by Cook, Fuente and Whittington}

Although there is an extensive literature on "pro-poor" water policies in low- and middle-income countries, studies have predominately focused on the question of whether increasing-block tariffs (IBTs) can effectively help the poor. Despite over two decades of evidence showing that "untargeted" IBTs often do a poor job of delivering subsidies to the poor (in large part because they are less likely to have piped connections), these tariff structures remain widespread. As discussed above, attention has recently turned in wealthier countries to using "non-tariff" customer assistance programs, or CAPs. These include various forms of consumption subsidies, flexible bill payment options, crisis assistance programs, and conservation assistance. How might these types of CAPs be implemented in low- and middleincome countries? When is it advisable for governments and utilities to focus on subsidies to connect households or subsidized non-network (e.g. kiosks) provision versus subsidies to connected customers? What are feasible targeting mechanisms to identify the poor? How can programs be financed? Cook et al. provide up-todate practical advice that water utilities, municipalities, welfare ministries, planning agencies, and donors can use to design and implement pro-poor water policies in low- and middle-income countries.

The authors begin with a set of questions aimed at identifying important contextual factors. They group these broadly into those that affect access to services and those that affect resources available for CAPs. Examples of factors affecting access include not only the percent connected to the piped network, but also the key reasons some households remain unconnected, the availability of public water sources (kiosks, fountains), and the prevalence of vended water and market prices for the service. Factors affecting resources available include general economic development, the existence of other social assistance programs, the technological capacity to implement subsidies using smartphone and prepaid kiosks, the availability of water resources, and the administrative capacity of the entity that would administer a CAP, including its ability to target the poor.

Cook et al. then review the characteristics of pro-poor policy options. These include how subsidies are administered, how they are funded, how poor households are targeted, and how subsidies are actually delivered (connection subsidies, 
subsidies for non-network water, monthly bill subsidies for connected households, etc.). Finally, the authors use information from these two sections in a simple typology of three illustrative cases that vary in terms of (1) the percentage of the population considered poor and (2) the percent of poor households who are connected to the piped network. They use this typology to highlight potential policy mistakes, discuss the challenges that a government or service provider is likely to face, and recommend policy changes.

\section{Synthesis and Policy Implications}

In summary, the articles in this special issue demonstrate many of the challenges in assessing how to help the poor afford reliable, high quality water supply and sanitation services. The papers by Hoque and Hope, Andres et al., and Thuy et al. all demonstrate why the current methods of assessing affordability are flawed, particularly for countries in the Global South. Hoque and Hope point out that affordability calculations focused only on prices will miss the fact that rural households make dynamic decisions throughout the year about where to collect water from, with their options often varying substantially in quality. Andres et al. and Thuy et al. both point out the difficulties - even for urban customers - of assessing affordability based on threshold values. Data are often lacking, and capital costs for connecting to the network or coping with poor service are often ignored. It will be challenging for governments to manage "affordability" programs across multiple sectors contained in the SDGs, since income available for any individual sector depends on what is spent in every other sector. Vincent $e t$ al. point out that although payment for ecosystem services (PES) programs have been widely adopted and studied, we know relatively little about their impact on the poorest.

But the papers also point to new opportunities and answers. Hoque and Hope use "water diaries" to illustrate the complexity of rural water supply and advance the conversation on rural affordability. Andres et al. develop a "basket of services" approach that can be used to simulate affordability under a number of policy counter-factuals, disaggregating customers into those with and without access to high-quality services. Thuy et al. ask households directly about how they perceive the "reasonableness" of water bills, finding surprising support for the idea that households believe some bills are "too low". Pairing panel data on water utilities operating costs with land cover change, Vincent et al. find no evidence that forest protection disproportionately helps or harms the poor. Finally, Cook et al. marshals a comprehensive view of customer assistance programs used around the world and 
provides a typology that can help governments and service providers find the types of programs that best suit their situation.

\section{References}

Bouma, D (2018). Water Affordability based on Income: The Tiered Assistance Program in Philadelphia. Available at: http://graham.umich.edu/media/pubs/Water-CSPhilidelphia-Tiered-Assistant-Program_0.pdf.

Campbell-Ferrari, A and L Wilson (2020). The COVID-Water Disconnect: How Statewide Moratoriums Are Leaving People Behind.

Pierce, G et al. (2020). Recommendations for Implementation of a Statewide Low-Income Water Rate Assistance Program. doi: 10.1109/isscs.2005.1511257.

US EPA (2016). Drinking Water and Wastewater Utility Customer Assistance Programs. WAREG (2017). Affordability in European Water Systems. 